\title{
THE RELATION OF METHEMOGLOBIN TO THE CYANOSIS OBSERVED AFTER SULFANILAMIDE ADMINISTRATION
}

\author{
By IRWIN VIGNESS, C. J. WATSON, AND W. W. SPINK \\ (From the Divisions of Internal Medicine and Biophysics, University of Minnesota Hospital, \\ Minneapolis)
}

(Received for publication August 18, 1939)

We have stated in a preliminary report that the cyanosis observed in individuals treated with sulfanilamide is due to methemoglobin, and rarely, to sulfhemoglobin (1). We recognized at the time that attempts had been made to explain the presence of cyanosis on a different basis. Marshall and Walzl (2) maintained that a black pigment derived from sulfanilamide, and present in the blood, was the cause of this cyanosis. A similar explanation was advanced by Ottenberg and Fox (3). They described the phenomenon of a bluish-purple pigment resulting when a colorless sulfanilamide solution was exposed to ultra violet light. We have confirmed this photochemical change of sulfanilamide. The results of our investigation are in agreement with those of Hartmann and his associates who showed that the cyanosis was related to methemoglobin formation and that methylene blue administered to cyanotic patients caused a disappearance of the cyanosis and a reduction in the concentration of methemoglobin (4).

At this time we desire to present further evidence that the cyanosis is due solely to the presence of methemoglobin, and in rare instances, to sulfhemoglobin. We have also included the results of a study of individuals receiving sulfapyridine. Spectral distribution curves were obtained with the spectrophotometer, using normal human blood and the bloods of patients to whom sulfanilamide or sulfapyridine was being administered. In initiating such a study, we believed that if any pigment other than methemoglobin that might cause cyanosis were present, it would be detected in the spectral distribution curves.

\section{MATERIAL AND METHODS}

Spectroscopic studies have been carried out on the bloods of 3 normal adults, 15 patients receiving sulfanilamide, and 5 more to whom sulfapyridine was given. From 4 to 8 grams of sulfanilamide were administered to the patients daily in divided doses for several days because of the presence of an infection. The free sulfanilamide in their bloods was determined on the day the bloods were examined for methemoglobin. These levels ranged from 4.9 to $20.8 \mathrm{mgm}$. of sulfanilamide per $100 \mathrm{cc}$. of blood. Sulfapyridine was given in divided doses for a total of 4 to 9 grams per day. The blood levels of free sulfapyridine varied from 2 to $14 \mathrm{mgm}$. per $100 \mathrm{cc}$. of blood. Data for the absorption curves were obtained with a Marten's type polarization photometer in combination with a Bausch and Lomb spectrometer. The spectrophotometric examinations were made in the range from 480 to $700 \mathrm{~m} \mu$. Whole blood was diluted with distilled water to give a concentration of $1: 25$ or $1: 50$. This solution was centrifuged for 10 minutes at 2400 revolutions per minute (radius 10 inches) in order to remove the stroma of the cells and clarify the solution. Absorption cells 0.5 or $1.0 \mathrm{~cm}$. in length were used over the spectral range from 480 to $600 \mathrm{~m} \mu$, and $3 \mathrm{~cm}$. cells were used from 600 to $700 \mathrm{~m} \mu$. The slit of the spectrometer was opened fairly wide during these observations so that more light could be gathered. This reduced the height of the oxyhemoglobin absorption peak at $576 \mathrm{~m} \mu$ a few per cent below the values which would have been obtained with a narrow slit, due to the decreased resolving power. A constant slit width was used throughout.

The first step was to obtain the normal absorption spectra for oxyhemoglobin and methemoglobin. Bloods from normal individuals were used, and dilutions were made as previously mentioned with distilled water. Oxyhemoglobin and methemoglobin spectral distribution curves were made from absorption spectra of the same samples of blood. Absorption of oxyhemoglobin at $576 \mathrm{~m} \mu$ was arbitrarily made unity in order to cancel out the effect of concentration.

Methemoglobin was formed by the addition of an excess of potassium ferricyanide. (The amount was not measured, but approximated $100 \mathrm{mgm}$. per gram of hemoglobin.) In each instance it was readily established spectroscopically that the conversion to methemoglobin was complete.

\section{RESULTS}

Normal absorption curves for oxyhemoglobin and for methemoglobin are shown in Figure 1. These curves are the average of several that were obtained by noting the absorption of blood solutions from 3 normal individuals. From the 


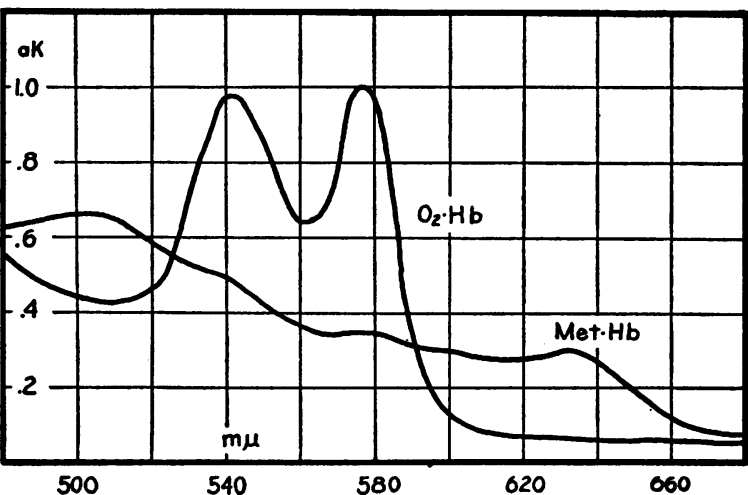

Fig. 1. Absorption Curves of Methemoglobin and OXYHEMOGLOBIN

Blood diluted with distilled water and centrifuged.

curves in Figure 1, it was found possible to construct curves representing bloods with given ratios of oxyhemoglobin and methemoglobin. These could then be used as a basis for a quantitative chemical analysis of blood containing methemoglobin, provided there were no other foreign colored substances present in the blood. In Figures 2 and 3 such a family of curves is shown for blood diluted with distilled water. In these figures, the values indicating the amount of absorption are made unity at $576 \mathrm{~m} \mu$ (Figure 2) and $630 \mathrm{~m} \mu$ (Figure 3 ) respectively. In other words, one could consider the values plotted to be the ratio of the absorption at any particular wavelength to the absorption of $576 \mathrm{~m} \mu$ for Figure 2, and correspondingly at $630 \mathrm{~m} \mu$ for

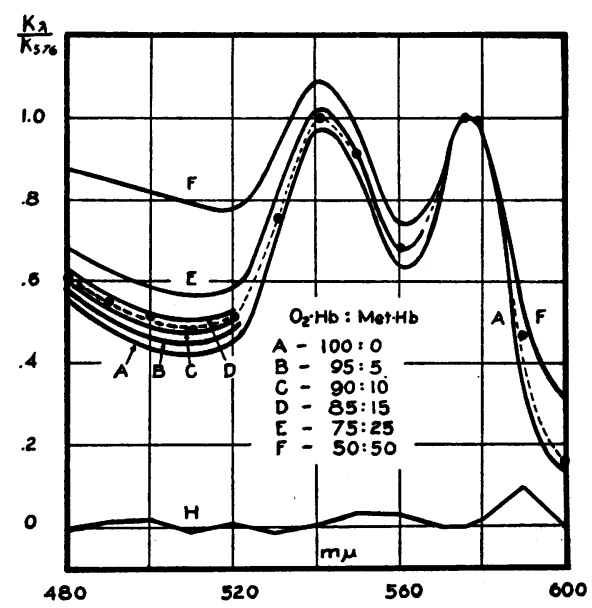

Fig. 2. Ratio of the Absorption at any Given Wavelength to the Absorption at 576m $\mu$ for Blood Containing Different Proportions of OxyhemoGLOBIN AND METHEMOGLOBIN

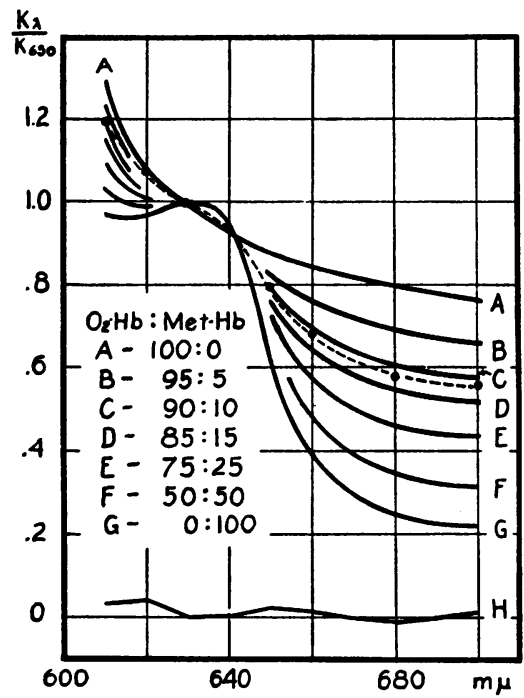

Fig. 3. Ratio of the Absorption at aNy Given

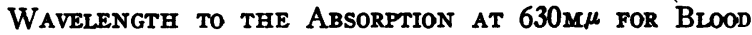
Containing Different Proportions of Oxyhemoglobin AND METhemoglobiN

Figure 3. In this manner, a correction is made for different concentrations of hemoglobin in various samples of blood. The characteristic absorption band of methemoglobin has a maximum at $630 \mathrm{~m} \mu$ in neutral or slightly acid solution, which makes its identification possible if other foreign colored substances were present in the blood. Therefore, absorption curves of blood containing methemoglobin should match one of the calculated curves of Figures 2 and 3 throughout the entire spectrum. Any other colored substance could then be detected by the divergence of these curves.

Blood was obtained from a patient who was moderately cyanotic due to sulfanilamide. Spectroscopic examination of the blood revealed the presence of an absorption band at $630 \mathrm{~m} \mu$, which is characteristic for methemoglobin. A spectrophotometric study of the same sample of blood was made, and the dotted curve seen in Figures 2 and 3 represents the absorption curve of this blood. From the position of this curve, it was estimated that this blood contained 12 per cent methemoglobin.

If the dotted curve in Figures 2 and 3 is subtracted from a theoretical curve that represents 12 per cent methemoglobin (not shown in figures), the curve of the difference is represented by $\mathrm{H}$. The absorption represented by $\mathrm{H}$ is no 
more than would be expected from experimental variations. Therefore, our observations are consistent with the interpretation that no colored substance is present possessing a significant amount of absorption over the wavelengths covered other than methemoglobin and oxyhemoglobin. The blood studied in this and other experiments reported in the paper was diluted with distilled water; similar results have been obtained with blood diluted in 0.4 per cent ammonia, although the absorption spectra were quite different. With a spectrocolorimetric method previously described, the value for the methemoglobin concentration for the sample of blood represented by the dotted curve was 10 per cent. In 7 additional instances compared, close correlation was found between the results of the spectrophotometric and spectrocolorimetric determinations. These data were tabulated in our previous report (1). Since the spectrocolorimetric method depends entirely upon the characteristic absorption band of methemoglobin at $630 \mathrm{~m} \mu$, it is unlikely that such a correlation would be found to exist if pigments other than methemoglobin and oxyhemoglobin were present in appreciable quantities. The possibility might be considered that another pigment having absorption at $630 \mathrm{~m} \mu$ was present, but it would be an unlikely coincidence that, in addition to possessing very similar absorption spectra, such absorption would also be completely removed by methylene blue. This is discussed later.

The amount of methemoglobin in a series of 13 determinations, as calculated in a manner similar to that described by Heilmeyer (5), is given in Table I. The determinations are made from the ratio of the extinction coefficients $K_{700} / K_{830}$ and $K_{510} / K_{578}$. The subscripts refer to the wavelength at which the extinction coefficient was determined.

Differences in the amount of methemoglobin as determined in the different parts of the spectrum, can be ascribed to an unknown pigment or to experimental errors. The majority of the determinations of this table have had their spectral distribution curves plotted in the manner illustrated in Figures 2 and 3 . In these cases, the curves followed those of a calculated methemoglobin concentration quite closely throughout. Thus it is believed that pigments of unknown nature
TABLE I

Per cent methemoglobin

\begin{tabular}{|c|c|c|c|c|c|c|c|c|c|c|c|c|c|}
\hline Number & 1 & 2 & 3 & 4 & 5 & 6 & 7 & 8 & 9 & 10 & 11 & 12 & 13 \\
\hline$K_{700} / K_{600}$ & 7.0 & 8.0 & 5.0 & 10.5 & 11.5 & 16.0 & 12.7 & 18.0 & 11.0 & 9.5 & 1.0 & 10.0 & 0.5 \\
\hline$K_{610} / K_{376}$ & 12.0 & 7.5 & 5.0 & 9.2 & 11.0 & 21.0 & 14.0 & 25.0 & 15.5 & 15.0 & 0.0 & 10.0 & 1.5 \\
\hline
\end{tabular}

are not present in sufficient quantity to contribute to the production of cyanosis. Determinations 11 and 13 in Table I were made on the bloods of patients who were not cyanotic.

A cyanotic patient, whose blood contained 12.3 per cent methemoglobin as determined from the absorption curves' calculation, was given intravenously $20 \mathrm{cc}$. of a 1 per cent solution of methylene blue. One-half hour after this injection the blood of the patient was examined with the spectrophotometer and the absorption spectrum obtained. This spectrum was that of normal blood (determination 11 of Table I). So it would appear that the methylene blue caused the removal of methemoglobin as well as other colored substances, if the latter were present. It is important to point out that the introduction of methylene blue into this patient's blood caused a decrease of about 20 per cent of the absorption due to oxyhemoglobin content during this half-hour period. This may well account for the temporary hyperpnea these patients exhibit shortly after the injection of methylene blue, usually lasting for 15 to 30 minutes.

The blood of another patient who was being treated with sulfanilamide was studied. He had no evidence of cyanosis at the time that his blood contained $13 \mathrm{mgm}$. per $100 \mathrm{cc}$. of free sulfanilamide. An absorption spectrum of his blood was obtained at this time showing an approximately normal spectrum. The methemoglobin concentration was about 1 per cent (determination 13, Table I).

It is of interest that over 50 patients have been observed while being treated with sulfapyridine. In but 1 of these patients has cyanosis been noted that could be attributed to the drug. This patient had a Type XXVI pneumococcus pneumonia involving the right lower lobe, with an associated massive collapse of the same lobe. Sulfapyridine was administered, and after 48 hours mild cyanosis was noted. At this time, spectroscopic examination of laked blood (1:5 with distilled 
water) revealed a very weak absorption band at $630 \mathrm{~m} \mu$ (maximum). When the same sample of blood was examined spectrophotometrically, the peak of the absorption curve was in the same range as for methemoglobin. One difference in the curve was noted. Absorption was slightly increased in the whole region of $600 \mathrm{~m} \mu$ to $700 \mathrm{~m} \mu$, as compared to the bloods from patients receiving sulfanilamide. With the spectrophotometric method described above, the value for methemoglobin (assuming that the total absorption was due only to met- and oxyhemoglobins) was 7 per cent. Thirty minutes after the patient had received $18 \mathrm{cc}$. of 1 per cent methylene blue solution intravenously, examination of the blood showed the normal absorption spectrum for oxyhemoglobin. The presence of methemoglobin in the blood of this individual, who received sulfapyridine, appears to be in agreement with the more extensive observations of Barnett and his associates (9).

The absorption spectra of the bloods of 3 other individuals receiving sulfapyridine were studied and were found to be quite close to normal, even when the blood contained as much as $5.4 \mathrm{mgm}$. of free sulfapyridine and $1 \mathrm{mgm}$. of the acetylated form per $100 \mathrm{cc}$. There was no methemoglobin absorption. In these spectral distribution curves there was a little greater absorption of quite uniform magnitude between 600 and $700 \mathrm{~m} \mu$ and for a given amount of hemoglobin, as measured by the $576 \mathrm{~m} \mu$ absorption peak, there was a slightly greater than normal absorption in the region around $500 \mathrm{~m} \mu$. This may indicate the presence of a small amount of an unknown pigment.

The absorption spectrum was determined for the bluish-purple pigment that results when dilute solutions of sulfanilamide are irradiated with ultra violet. This spectrum exhibits a broad absorption with a maximum at $570 \mathrm{~m} \mu$, from which point it decreases gradually throughout the visible spectrum. Its presence, when mixed with blood, would be detected by an increase in absorption in the red region of the spectrum. There should be no difficulty in separating methemoglobin from this pigment.

\section{DISCUSSION}

As noted in our preliminary report (1), the blood from patients who have become cyanotic after receiving sulfanilamide has always been found to contain methemoglobin (or rarely sulfhemoglobin) when examined in sufficient concentration. We believe that failure by others to observe methemoglobin in such instances is probably on the basis of too great dilution of the laked blood. Thus we have noticed on a number of occasions that the methemoglobin band could not be detected in blood diluted $1: 20$ or even $1: 10$ with distilled water, but that it was distinctly visible in a dilution of $1: 5$. (This applies to a 2 $\mathrm{cm}$. thickness with a Zeiss grating spectrometer). The present spectrophotometric study has failed to reveal the presence of any pigment other than methemoglobin in the blood of cyanotic patients receiving sulfanilamide. On the other hand, neither cyanosis nor methemoglobinemia has been noted following sulfapyridine therapy. In this connection, it is of interest that we have not been able to affect sulfapyridine with ultra violet light. Pigments such as those derived from sulfanilamide under similar conditions do not appear. The significance of this difference is not clear, but it may be that methemoglobin formation is dependent upon derivatives of sulfanilamide which are not formed in the body from sulfapyridine.

Webb and Kniazuk (6) have recently reported the presence of pigments other than met- or sulfhemoglobin (although these were also noted at times) in the blood of rats receiving sulfanilamide. In comparing their results with those of the present study, several points deserve mention. The difference in species may or may not be of significance. The amount of sulfanilamide which they used was 15 to 40 times that used in the patients of the present series. It would be interesting to know whether the concentration of sulfanilamide in the rats' blood was in any way correlated with Webb and Kniazuk's finding of pigments other than met- or sulfhemoglobin. They did not report data on the blood sulfanilamide level. The very large doses which they used evidently were often productive of severe toxic effects since they note that " when there seemed to be danger of the animal dying, the dose was reduced to 1.5 grams per kilo" (from 2 grams per kilo). Severe toxicity, on the basis of such massive dosage, is a factor of unknown significance with respect to pigment metabolism, and this factor was absent in the present study. 
Wendel and Wendel ( 7 ) have recently reported their results of spectrophotometric and gasometric measurements of the bloods from cyanotic patients receiving sulfanilamide. Their data indicate that methemoglobin is the principal if not the sole source of the abnormal color of the blood. On the other hand, Bigler and Werner (8), using a gasometric method, found that appreciable cyanosis may occur without the presence of inactive hemoglobin. Likewise, cyanosis occurred without methemoglobin or sulfhemoglobin being demonstrated. It is difficult to interpret the results of Bigler and Werner, since they do not state the dilution of blood examined spectroscopically or the thickness of the tube used.

\section{SUM MARY AND CONCLUSIONS}

1. The cyanosis observed in man following sulfanilamide therapy is explained by the presence of methemoglobin (rarely sulfhemoglobin).

2. Methylene blue abolishes the cyanosis due to methemoglobin (and other pigments if present, except sulfhemoglobin), and the spectral distribution curve of the blood becomes normal.

3. Spectrophotometric studies of the blood of sulfanilamide-treated patients have failed to reveal the presence of pigments other than methemo- globin in quantities large enough to contribute appreciably to cyanosis.

\section{BIBLIOGRAPHY}

1. Watson, C. J., Vigness, I., and Spink, W. W., Relation of methemoglobin to the cyanosis observed after sulfanilamide administration. Proc. Soc. Exper. Biol. and Med., 1939, 40, 547.

2. Marshall, E. K., Jr., and Walzl, E. M., On the cyanosis from sulphanilamide. Bull. Johns Hopkins Hosp., 1937, 61, 140.

3. Ottenberg, R., and Fox, C. L., Jr., Explanation for the cyanosis of sulfanilamide therapy. Proc. Soc. Exper. Biol. and Med., 1938, 38, 479.

4. Hartmann, A. F., Perley, A. M., and Barnett, H. L., A study of some of the physiological effects of sulfanilamide. II. Methemoglobin formation and its control, J. Clin. Invest., 1938, 17, 699.

5. Heilmeyer, L., Medizinische Spektrophotometrie. Jena, G. Fischer, 1933, p. 103.

6. Webb, T. J., and Kniazuk, M., Spectrophotometric examination of blood from animals receiving sulfanilamide. J. Biol. Chem., 1939, 128, 511.

7. Wendel, W. B., and Wendel, N. M., Nature of the abnormal pigment in the blood of individuals receiving sulfanilamide treatment. Proc. of the Amer. Soc. of Biol. Chem., J. Biol. Chem., 1939, 128, 109.

8. Bigler, J. A., and Werner, M., Cyanosis from use of sulfanilamide. Am. J. Dis. Child., 1939, 57, 1338.

9. Barnett, H. L., Hartmann, A. F., Perley, A. M., and Ruhoff, M. B., The treatment of pneumococcic infections in infants and children with sulfapyridine. J. A. M. A., 1939, 112, 518. 